\title{
On Newton's method applied to real polynomials
}

\author{
Figen ÇiLINGiR * \\ Dept. of Mathematics and Statistics \\ Boston University \\ 111 Cummington Street, 02215 Boston, MA \\ USA \\ cilingir@bu.edu, cilingirfigen@gmail.com
}

\author{
Xavier JARQUE ${ }^{\dagger}$ \\ Dept. Enginyeria Informàtica i Matemàtiques \\ Universitat Rovira i Virgili \\ Avinguda Països Catalans, 26 \\ Campus Sescelades, 43007 Tarragona \\ Catalunya, Spain \\ xavier.jarque@urv.cat
}

March 17, 2010

\begin{abstract}
It is known that if we apply Newton's method to the complex function $F(z)=P(z) e^{Q(z)}$, with $\operatorname{deg}(Q)>2$, then the immediate basin of attraction of the roots of $P$ has finite area. In this paper we show that under certain conditions on $P$, if $\operatorname{deg}(Q)=1$, then there is at least one immediate basin of attraction having infinite area.
\end{abstract}

\section{Introduction}

Newton's method (in one variable) is an iterative algorithm for finding the zeros of a holomorphic or meromorphic function $f(z)$, or equivalently, for solving the equation $f(z)=0$. Newton's method applied to $f$, usually denote by $N_{f}$, starts with an initial condition $z_{0} \in \mathbb{C}$ and computes recursively $z_{n+1}$ as

$$
z_{n+1}=N_{f}\left(z_{n}\right):=z_{n}-\frac{f\left(z_{n}\right)}{f^{\prime}\left(z_{n}\right)} .
$$

This method works since, if $z_{0}$ is chosen close enough to a zero $\zeta$ of $f$ (that is $\zeta$ satisfies $f(\zeta)=0)$, then

$$
N_{f}\left(z_{n}\right) \rightarrow \zeta, \text { as } n \rightarrow \infty .
$$

One thinks Newton's method as a dynamical system, this property is equivalent to saying that all (finite) zeros of $f$ are attracting fixed points of $N_{f}$. Moreover, and this is a key point, $N_{f}$ has no other finite fixed points.

${ }^{*}$ The first author would like to thank TUBITTAK for their support while this research was in progress.

${ }^{\dagger}$ The second author is partially supported by MTM2006-05849 and MTM-2008-01486/Consolider (including a FEDER contribution) and by 2005SGR-00550. He would also like to thank the Ministerio de Ciencia y Innovacion of Spain for the financial support when visiting Boston University. 
From the numerical point of view, Newton's method is very useful (quadratic convergence to all simple roots, simplicity, etc) and so it has became the universal method to compute zeroes of functions. However, it has some limitations. For instance it is known that for certain functions (even for polynomial) there are open sets in $\mathbb{C}$ such that if the initial condition is chosen in those sets the iterative algorithm does not converge to any of the zeroes of $f$. Another obstruction is that, a priori, the method does not tell you where to chose the initial conditions so that you get all of the roots of $f$ (see [7] where the authors show, among many other interesting results, how to choose the initial conditions in an efficient way when $f$ is polynomial).

Newton's method has been applied and studied (as a numerical method and as a dynamical system) for different kinds of families of functions but the most well known case is the polynomial case, $f:=P$. Indeed the study of Newton's method applied to degree 2 and degree 3 polynomials was the germ of what we known today as complex dynamics. Notice that $N_{P}$ is a rational map and, consequently the Newton's method is a dynamical system defined in $\mathbb{C}_{\infty}=\mathbb{C} \cup \infty$. It is an exercise to see that $z=\infty$ is a repelling fixed point for $N_{P}$.

In this paper, we consider Newton's method applied to the transcendental entire function $F(z)=P(z) e^{Q(z)}$ where $P$ and $Q$ are polynomials. Since the exponential map is always different from zero, we still have the condition that the roots of $P$ correspond to attracting fixed points of $N_{F}$ and vice versa. Moreover, it is easy to see that even though $F$ is entire, $N_{F}$ is a rational map. However, one main distinction occurs at infinity since now $\infty$ is a parabolic fixed point for $N_{F}$ instead of a repelling fixed point for $N_{P}$.

The main reason to introducing the transcendental entire ingredient is twofold. On the one hand we would like to explore if, as a numerical method for finding the roots of $P$, it presents some advantages, and on the other hand we want to see its properties as a dynamical system defined in $\mathbb{C}_{\infty}$. A primer answer to those questions is due to M. Haruta, who proved in [5] that if the degree of the polynomial $Q$ is greater than 2 then the area of the immediate basin of attraction of all fixed points of $N_{F}$ is finite (see also [2,3] for an extension to relaxed Newton's method). From the numerical point of view this can be understood as a negative result. It was also shown, as a corollary of the Fatou's Flower Theorem, that the number of attracting petals of $\infty$ coincides with the degree of $Q$.

The condition $\operatorname{deg}(Q) \geq 3$ it is essential to show the finiteness of the area, and the arguments do not apply to $Q$ with lower degree. Indeed, the question of the finiteness of the area of the immediate basins of attraction of the fixed points of $N_{F}$ when the degree of $Q$ is less than 3, is still open in the general case (arbitrary $P$ ). As a partial result in this direction, it was shown in [4] that for $F(z)=z e^{z}$ the immediate basin of attraction of $z=0$ has infinite area. This result (added to a strong control on the distribution of the immediate basins of attraction) strengthens the idea that keeping $Q(z)=z$ instead of using higher degree polynomial is a good choice from the numerical point of view.

A first step though is to see if this area property is true for a general polynomials. In this paper we prove the following result:

Theorem A. Let $P(z)=z^{k} P_{1}(z), k \geq 1$ be a polynomial of degree $m \geq 2$ with real coefficients. Assume that for all $x>0$ we have $P_{1}(x)\left(P_{1}(x)+P_{1}^{\prime}(x)\right)>0$. Let $N_{F}(z)$ to be the Newton's method applied to the function $F(z)=P(z) e^{z}$. Then $N_{F}$ has 0 as an attracting fixed point whose immediate basin of attraction has infinite area.

As a corollary we have the following result. 
Corollary 1.1. Let $F(z)=P(z) e^{z}$ where $P$ is a polynomial of degree $m \geq 2$ with real coefficients and at least one real root. Denote by $x_{0}$ its largest real root. Then

(a) The linear change of variables $z \rightarrow z-x_{0}$ transforms $F(z)$ into $G(z):=F\left(z+x_{0}\right)=$ $z^{k} P_{1}(z) e^{z}$ for some $P_{1}$ with real coefficients, and such that $P_{1}(x) \neq 0$ for all $x \geq 0$.

(b) In the notation of $(a)$, if $P_{1}(x)\left(P_{1}(x)+P_{1}^{\prime}(x)\right)>0$ for all $x \geq 0$, then $N_{F}$ has $x_{0}$ as an attracting fixed point and its immediate basin of attraction has infinite area.

Before proving Theorem A (and the above corollary) in Section 4, we will give in Section 3 some numerical simulations and the corresponding pictures showing the shape and distribution of the (immediate) basins of attraction associated to the Newton's method applied to $F(z)=$ $P(z) e^{z}$. In Section 2 we give some preliminaries.

We want to thank Jordi Taixés for his help making the pictures of the paper.

\section{Preliminaries}

A rational map $R$ is a holomorphic function of the Riemann sphere $\mathbb{C}_{\infty}$ to itself, and it can be always expressed as $R=H / G$, where $H$ and $G$ are complex polynomials without a common divisor. Its degree is defined by $\operatorname{deg}(R)=\max \{\operatorname{deg}(H), \operatorname{deg}(G)\}$. We are interested in holomorphic dynamical systems that arise by iterating rational maps of degree $\geq 2$ on the Riemann sphere.

A point $\zeta \in \mathbb{C}$ is called a periodic point of period $n$ if $R^{n}(\zeta)=\zeta$ and $R^{k}(\zeta) \neq \zeta$ for all $k<n$, where $k, n \in \mathbb{N}$. If $n=1$ we say that $\zeta$ is a fixed point. The periodic point $\zeta$ is called attracting (respectively repelling) if $0<\left|\left(R^{n}\right)^{\prime}(\zeta)\right|<1$ (respectively $\left|\left(R^{n}\right)^{\prime}(\zeta)\right|>1$ ). If $\left(R^{n}\right)^{\prime}(\zeta)=0$ we say that $\zeta$ is superattracting. Finally, if $\left(R^{n}\right)^{\prime}(\zeta)=e^{2 \pi i t}, t \in \mathbb{R}$, we say that $\zeta$ is parabolic when $t \in \mathbb{Q}$ and irrationally indifferent when $t \in \mathbb{R} \backslash \mathbb{Q}$. Even though in rational iteration $z=\infty$ is a regular point (it cannot be distinguished from any other point of the plane), to compute the derivative at $\zeta=\infty$ in the above definitions we need first to conjugate the map by $z \rightarrow 1 / z$ and then consider $\zeta=0$.

The Julia set, denoted by $\mathcal{J}(R)$, can be defined as the closure of the set of repelling periodic points of $R$, and $\mathcal{J}(R)$ also coincides with the set of points in $\mathbb{C}_{\infty}$ for which the family of iterates $\left\{R^{n}\right\}_{n \in \mathbb{N}}$ fails to be a normal family (in the sense of Montel). The Fatou set, denote by $\mathcal{F}(R)$, is defined as the complement of $\mathcal{J}(R)$ so $\mathcal{F}(R)=\mathbb{C}_{\infty} \backslash \mathcal{J}(R)$. It is well known that $\mathcal{J}(R) \neq \emptyset$ and that the Fatou set is the union of open domains of the plane each of which is called a Fatou component. In particular, if $\zeta$ is an attracting fixed point of $R$, we define its basin of attraction as $A(\zeta):=\left\{z \in \mathbb{C} \mid R^{n}(z) \rightarrow \zeta, n \rightarrow \infty\right\}$. Each connected component of this set belongs to the Fatou set. The one which contains $z_{0}$ is called the immediate basin of attraction and is denoted by $A^{\star}(\zeta)$. (see [1] as a complete reference for rational iteration).

As for Newton's method applied to a polynomial $P$, it turns out that Newton's method applied to the entire transcendental map $F(z)=P(z) e^{z}$ is also a rational map. More precisely

$$
N_{F}(z)=z-\frac{P(z)}{P^{\prime}(z)+P(z)}=\frac{z\left(P^{\prime}(z)+P(z)\right)-P(z)}{P^{\prime}(z)+P(z)} .
$$

It is straightforward to see that $\zeta$ is a (simple) root of $P$ if and only if $\zeta$ is a (super)attracting fixed point of $N_{F}$. Since $N_{F}$ is rational, we conclude that $\infty$ has to be a weakly repelling fixed point, see for instance Corollary 12.7 in [6]. However, in contrast to Newton's method applied 
to a polynomial $P$, the point at infinity is not a repelling fixed point anymore. The following result is a corollary of Propositions 1 and 2 in [5].

Proposition 2.1. Infinity is a parabolic fixed point (with $t=0$ ) with a unique attracting (and repelling) petal.

Finally we state an important result proved by M. Shishikura in [8] that holds for any Newton's method with a rational expression.

Proposition 2.2. All Fatou components of $N_{F}$ are simply connected.

\section{Numerical simulations and examples of Theorem A}

In this section we provide some numerical experiments which exemplified the shape and distribution of the basins of attraction of the superattracting fixed points of $N_{F}$ for different polynomials. From those examples we can see some differences between the shape and distribution of the basins of attraction with respect to the standard Newton's method $N_{P}$. The simulations suggest that the area of all immediate basins of all attracting fixed points of $N_{F}$ will be infinite, but the arguments we use here to prove Theorem A cannot be extended straightforward.

To do so, we consider three different polynomials of degree 5. Each one will describe different scenarios in terms of the hypotheses of Theorem A (and Corollary 1.1). Precisely, let

$$
\begin{aligned}
& p(z)=5+11 z+7 z^{2}+2 z^{3}+2 z^{4}+z^{5}, \\
& q(z)=2.8125+6.3125 z+8 z^{2}+6.5 z^{3}+3 z^{4}+z^{5}, \quad \text { and } \\
& r(z)=-165.62+301.21 z-217.6 z^{2}+78.2 z^{3}-14 z^{4}+z^{5} .
\end{aligned}
$$

The zeros of $p$ are given by $z_{0} \approx-1.516, z_{1}=-1$ (double), $z_{2} \approx 0.758-1.65 i$ and $z_{3}=\overline{z_{2}}$. So if we apply the linear change of variables $z \rightarrow z+1$ the polynomial $p$ is transformed into the polynomial

$$
\tilde{p}(z)=z^{2}\left(3+4 z-3 z^{2}+z^{3}\right),
$$

or, equivalently, $\tilde{p}(z)=z^{2} P_{1}(z)$ with $P_{1}(z)=3+4 z-3 z^{2}+z^{3}$. One can check that $P_{1}(x)\left(P_{1}(x)+P_{1}^{\prime}(x)\right)>0$ for all $x>0$. Thus statement (b) of Corollary 1.1 concludes that the immediate basin of attraction of $z_{1}=-1$ (i.e., the largest real root of the polynomial $p$ ) of Newton's method applied to $F(z)=p(z) e^{z}$ has infinite area. In Figure 1 we illustrate the shape and distribution of the immediate basins of attraction of the attracting fixed points of $N_{G}$, where $G(z)=z^{2} P_{1}(z) e^{z}$, on the (dynamical) plane as well as on the Riemann sphere.

The zeros of $q$ are given by $z_{0}=-1, z_{1}=-0.5+i, z_{2}=\overline{z_{1}}, z_{3}=-0.5+\sqrt{2} i$ and $z_{4}=\overline{z_{3}}$. So, if we apply the linear change of variables $z \rightarrow z+1$ the polynomial $q$ is transformed into the polynomial

$$
\tilde{q}(z)=z\left(2.8125-3.5 z+4.5 z^{2}-2 z^{3}+z^{4}\right),
$$

or, equivalently, $\tilde{q}(z)=z Q_{1}(z)$, where $Q_{1}(z)=2.8125-3.5 z+4.5 z^{2}-2 z^{3}+z^{4}$. One can easily check that $Q_{1}(x)>0$ but $Q_{1}(x)+Q_{1}^{\prime}(x)$ is not strictly positive for all $x>0$, so Corollary 1.1 cannot be directly applied. The reason is that, to conclude from Lemma 4.1 that the positive real line belongs to the immediate basin of attraction of 0 for $N_{G}, G(z)=z^{k} P_{1}(z) e^{z}$, we use that $P_{1}(x)\left(P_{1}(x)+P_{1}^{\prime}(x)\right)>0$. However one can easily check for this concrete example that, even though the condition is not satisfied, the positive real line does belong to the immediate basin 


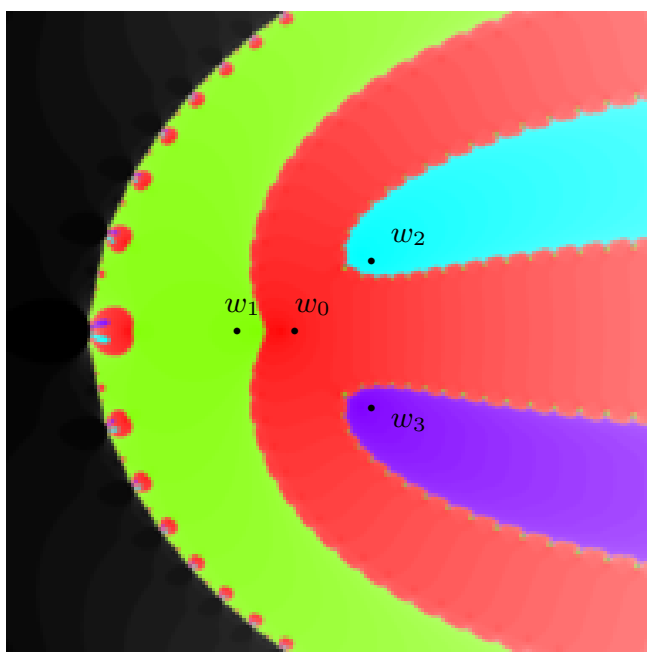

(a) Dynamical plane.

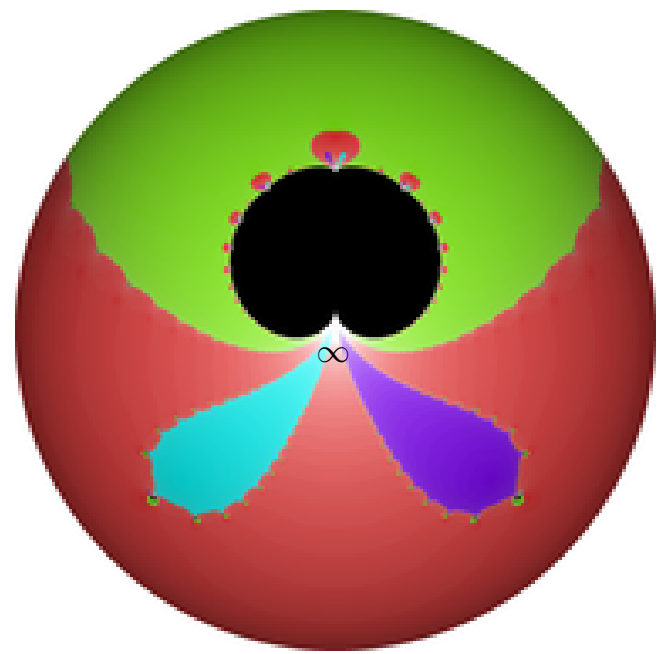

(b) Riemann sphere.

Figure 1: The pictures show the Newton 's method applied to $G(z)=z^{2} P_{1}(z) e^{z}$ with $P_{1}(z)=3+4 z-3 z^{2}+$ $z^{3}$. Each color corresponds to the basin of attraction of each attracting fixed point at $w_{0}=0, w_{1} \approx-0.516$, $w_{2} \approx 1.756-1.65 i$ and $w_{3}=\overline{w_{2}}$. In black we draw the basin of the parabolic fixed point at infinity. The little decorations of the four immediate basins correspond to their preimatges.

of attraction of 0 . Since this is the only step in the proof of Theorem A where this condition is used we can ensure that the immediate basin of attraction of $z_{1}=-1$ of Newton's method applied to $F(z)=q(z) e^{z}$ has infinite area (see also Remark 4.1). In Figure 2 we illustrate the shape and distribution of the immediate basins of attraction of the attracting fixed points of $N_{G}$, where $G(z)=z Q_{1}(z) e^{z}$, on the (dynamical) plane as well as on the Riemann sphere.

To finish this section we deal with the polynomial $r$. The zeros of $r$ are given by $z_{0}=2$, $z_{1} \approx 3+0.316 i$ (double) and $z_{2}=\bar{z}_{1}$ (double). So if we apply the linear change of variables $z \rightarrow z-2$ we get

$$
\tilde{r}(z)=z\left(1.21-4.4 z+6.2 z^{2}-4 z^{3}+z^{4}\right)
$$

or equivalently, $\tilde{r}(z)=z R_{1}(z)$, where $R_{1}(z)=1.21-4.4 z+6.2 z^{2}-4 z^{3}+z^{4}$. One can check that $R_{1}(x)>0$ but $R_{1}(x)+R_{1}^{\prime}(x)$ is not strictly positive for all $x>0$, and moreover $x\left(R_{1}(x)+R_{1}^{\prime}(x)\right)+R_{1}(x)$ changes sign for some $x>0$. As a consequence we can check that Newton's method applied to $G(z)=z R_{1}(z) e^{z}$ does not have the positive real line inside any of the immediate basins of attraction and so the arguments of the proof of the Theorem A will not be true anymore (see Remark 4.1). However, as we did in the previous two cases we draw in Figure 3 the shape and distribution of the immediate basins of attraction of the attracting fixed points of $N_{G}$, where $G(z)=z R_{1}(z) e^{z}$, on the (dynamical) plane as well as on the Riemann sphere. 


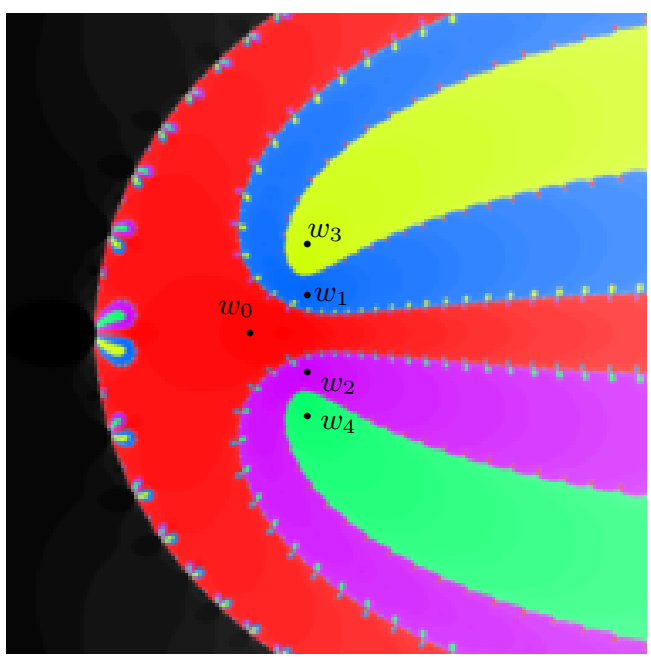

(a) Dynamical plane.

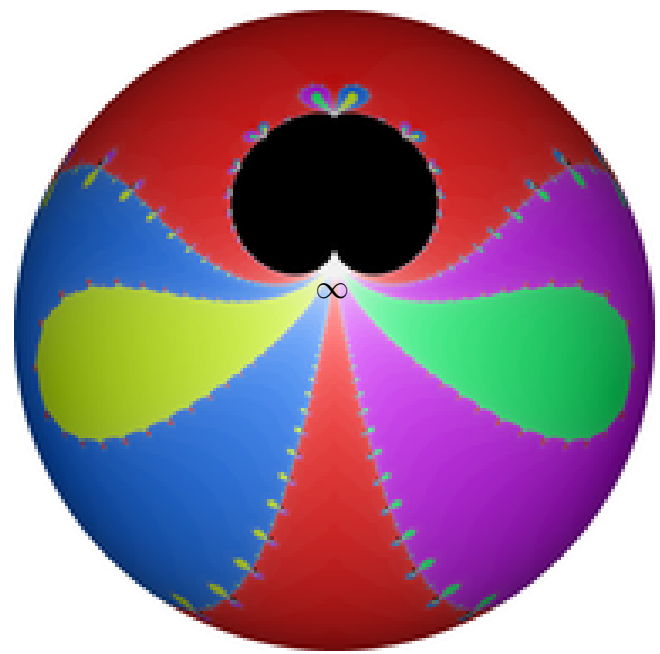

(b) Riemann sphere.

Figure 2: The pictures show the Newton 's method applied to $G(z)=z Q_{1}(z) e^{z}$ with $Q_{1}(z)=2.8125$ $3.5 z+4.5 z^{2}-2 z^{3}+z^{4}$. Each color corresponds to the basin of attraction of each attracting fixed points at $w_{0}=0, w_{1}=0.5+\sqrt{2} i, w_{2}=\overline{w_{1}}, w_{3}=0.5+i$ and $w_{4}=\overline{w_{3}}$. In black we draw the basin of the parabolic fixed point at infinity. The little decorations of the five immediate basins correspond to their preimatges.

\section{Proof of Theorem A}

We prove first Theorem A and then we conclude Corollary 1.1.

Lemma 4.1. Assume $P$ and $P_{1}$ as in Theorem $A$. Then if $F(z)=P(z) e^{z}$ the real function $N_{F}(x)$ is such that

(a) $N_{F}(0)=0, N_{F}^{\prime}(0)=1-\frac{1}{k}$.

(b) $N_{F}(x)$ tends to infinity and is asymptotic to $x-1$.

(c) $0<N_{F}(x)<x$, with no poles, for all $x>0$.

As a consequence, the positive real axis lies in the immediate basin of attraction of $z=0$ for $N_{F}$.

Proof. Since $P(z)=z^{k} P_{1}(z)$ we have

$$
N_{F}(z)=z-\frac{z P_{1}(z)}{z\left(P_{1}(z)+P_{1}^{\prime}(z)\right)+k P_{1}(z)}=z\left(\frac{z\left(P_{1}(z)+P_{1}^{\prime}(z)\right)+(k-1) P_{1}(z)}{z\left(P_{1}(z)+P_{1}^{\prime}(z)\right)+k P_{1}(z)}\right) .
$$

Statement (a) is a straightforward computation. From (2) we have

$$
N_{F}(x)=x\left(\frac{x\left(P_{1}(x)+P_{1}^{\prime}(x)\right)+(k-1) P_{1}(x)}{x\left(P_{1}(x)+P_{1}^{\prime}(x)\right)+k P_{1}(x)}\right) .
$$




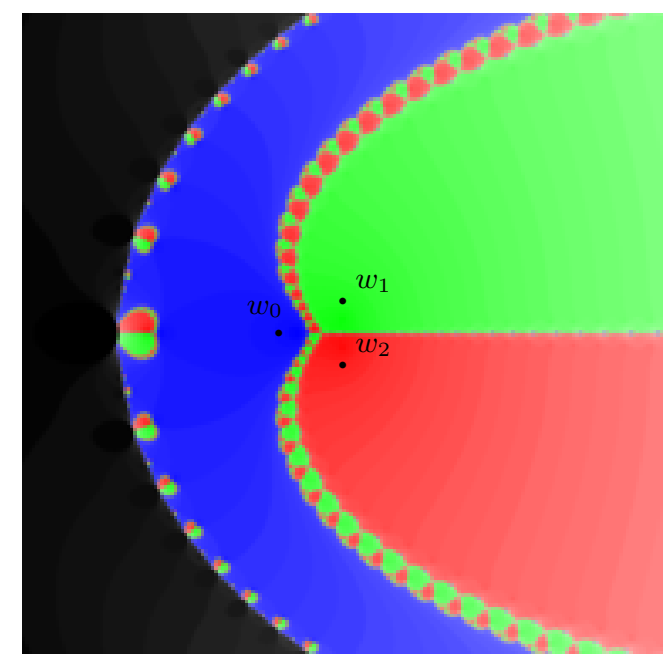

(a) Dynamical plane

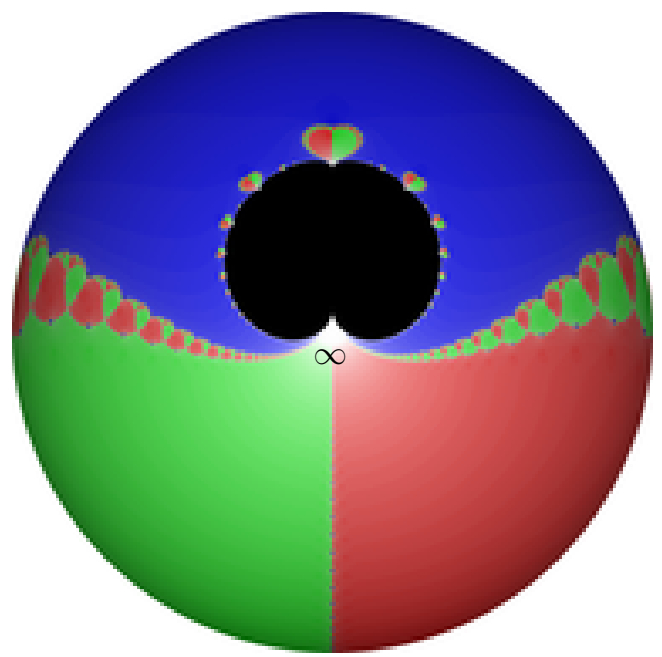

(b) Riemann sphere

Figure 3: The pictures show the Newton 's method applied to $G(z)=z R_{1}(z) e^{z}$ with $R_{1}(z)=1.21-4.4 z+$ $6.2 z^{2}-4 z^{3}+z^{4}$. Each color corresponds to the basin of attraction of each attracting fixed point at $w_{0}=0$, $w_{1} \approx 1+0.316 i$, and $w_{2}=\overline{w_{1}}\left(w_{1}\right.$ as well as $w_{2}$ are double roots of $\left.R_{1}\right)$. Notice that the positive real line is not included in the basin of attraction of $w=0$ and so Theorem A does not apply in this case (see Lemma 4.3 and Remark 4.1), although it seems that the area is infinite as well. In black we draw the basin of the parabolic fixed point at infinity. The little decorations of the three immediate basins correspond to their preimatges.

Thus it is easy to verify that

$$
\lim _{x \rightarrow \infty} \frac{N_{F}(x)}{x}=1 \quad \text { and } \quad \lim _{x \rightarrow \infty} x-N_{F}(x)=1,
$$

and statement (b) is proved. Finally, since $P_{1}(x)\left(P_{1}(x)+P_{1}^{\prime}(x)\right)>0$ for all $x>0$, we have that the factor in parenthesis in the previous expression of $N_{F}(x)$ is between 0 and 1 for all $x>0$, and so $0<N_{F}(x)<x$ for all $x>0$. Thus (c) follows and the positive real axis lies in the immediate basin of attraction of $z=0$ for $N_{F}$.

Remark 4.1. The (sufficient) condition $P_{1}(x)\left(P_{1}(x)+P_{1}^{\prime}(x)\right)>0$ for all $x>0$ in Theorem $A$ is only used when proving Lemma 4.1(c). We remark that a better (although still not sharped) sufficient condition in order to prove Lemma 4.1(c) (and consequently to prove Theorem A) would be

$$
P_{1}(x)\left[x\left(P_{1}(x)+P_{1}^{\prime}(x)\right)+(k-1) P_{1}(x)\right]>0 .
$$

Once we know that the positive real line lies in the immediate basin of attraction of $z=0$ for $N_{F}$ we will show that there is an $\varepsilon$ neighborhood of $\mathbb{R}^{+}$that also lies inside $A^{\star}(0)$. More precisely, we will show that if we define $A_{r}^{\varepsilon}=\{z=x+i y|x \geq r| y \mid,<\varepsilon\}$ then $A_{0}^{\varepsilon} \subset A^{\star}(0)$, for $\varepsilon$ sufficiently small. Since $A_{0}^{\varepsilon}$ has infinite area for all positive $\varepsilon$ this will imply Theorem A. 
To simplify the presentation we define

$$
R(z)=\frac{P(z)}{P(z)+P^{\prime}(z)}
$$

so that equation (1) may be written as

$$
N_{F}(z)=z-R(z) .
$$

Lemma 4.2. Let $z=x+y i$ and $p(z)=a_{0}+a_{1} z+\ldots a_{n} z^{n}$. Let us write $p(x+y i)=\alpha+\beta i$ and $p^{\prime}(x+y i)=\zeta+\delta i$. Then

$$
\begin{aligned}
\alpha & =A_{0}(y)+A_{1}(y) x+\ldots+A_{n-1}(y) x^{n-1}+a_{n} x^{n}, \\
\beta & =y\left[B_{0}(y)+B_{1}(y) x+\ldots+B_{n-2}(y) x^{n-2}+n a_{n} x^{n-1}\right], \\
\zeta & =C_{0}(y)+C_{1}(y) x+\ldots+C_{n-2}(y) x^{n-2}+n a_{n} x^{n-1}, \\
\delta & =y\left[D_{0}(y)+D_{1}(y) x+\ldots+D_{n-3}(y) x^{n-3}+n(n-1) a_{n} x^{n-2}\right],
\end{aligned}
$$

where $A_{i}(y), B_{i}(y), C_{i}(y), D_{i}(y)$ are polynomials in the variable $y$ with coefficients given by the coefficients of $p$.

Proof. Substituting $z=x+y i$ in the expression of $p(z)$ we have

$$
\begin{aligned}
p(x+y i) & =a_{0}+a_{1}(x+y i)+a_{2}(x+y i)^{2}+\ldots+a_{n}(x+y i)^{n}= \\
& =a_{0}+a_{1}(x+y i)+\sum_{k=0}^{2}\left(\begin{array}{l}
2 \\
k
\end{array}\right) x^{2-k}(y i)^{k}+\ldots+\sum_{k=0}^{n}\left(\begin{array}{c}
n \\
k
\end{array}\right) x^{n-k}(y i)^{k} .
\end{aligned}
$$

Taking real and imaginary part, and expanding in the variable $x$ we get the expressions for $\alpha$ and $\beta$ given in (5). In a similar way we deduce the corresponding expressions for $\zeta$ and $\delta$.

Lemma 4.3. Let $z=x+y i$. The following statements hold

(a) $\operatorname{Re}\left(N_{F}(z)\right)=x-\operatorname{Re}(R(z))$, with

$$
\operatorname{Re}(R(z))=\frac{\alpha(\alpha+\zeta)+\beta(\beta+\delta)}{(\alpha+\zeta)^{2}+(\beta+\delta)^{2}}
$$

(b) $\operatorname{Im}\left(N_{F}(z)\right)=y-\operatorname{Im}(R(z))$, with

$$
\operatorname{Im}(R(z))=\frac{\beta \zeta-\alpha \delta}{(\alpha+\zeta)^{2}+(\beta+\delta)^{2}}
$$

Proof. From (3) we deduce that

$$
N_{F}(x+y i)=(x+y i)-\frac{(\alpha+\beta i)}{(\alpha+\beta i)+(\zeta+\delta i)},
$$

where $\alpha, \beta, \zeta, \delta$ are defined in Lemma 4.2. Doing some computations and taking real and imaginary part of this equality we end up with the expressions of $\operatorname{Re}(R(z))$ and $\operatorname{Im}(R(z))$ given by (6) and (7). 
Lemma 4.4. For $r>0$ sufficiently large, $N_{F}\left(A_{r+2}^{\varepsilon}\right) \subset A_{r}^{\varepsilon}$ for all $\varepsilon \in(0,1)$.

Proof. We claim that

$$
\lim _{x \rightarrow \infty,|y|<1} \operatorname{Re}(R(z))=1 \quad \text { and } \quad \lim _{x \rightarrow \infty,|y|<1} \operatorname{Im}(R(z))=0 .
$$

The claim follows by noticing that from (5), (6) and (7) we have that

$$
\operatorname{Re}(R(z))=\frac{a_{n}^{2} x^{2 n}+\tilde{o}\left(x^{2 n+2}\right)}{a_{n}^{2} x^{2 n}+\tilde{o}\left(x^{2 n}\right)} \text { and } \operatorname{Im}(R(z))=y\left[\frac{n a_{n}^{2} x^{2 n-2}+\tilde{o}\left(x^{2 n-2}\right)}{a_{n}^{2} x^{2 n}+\tilde{o}\left(x^{2 n}\right)}\right]
$$

where

$$
\lim _{x \rightarrow \infty} \frac{\tilde{o}\left(x^{k}\right)}{x^{k}}=0, k>0,
$$

uniformly in $y$ for $|y| \leq 1$. On one hand from (8) we can find $r_{1}$ so that

$$
\operatorname{Re}\left(N_{F}(z)\right)=\operatorname{Re}(z)-\operatorname{Re}(R(z))<\operatorname{Re}(z)-0.5
$$

for all $z \in A_{r_{1}}^{\varepsilon}$. On the other hand, from (9), we have that

$$
\operatorname{Im}\left(N_{F}(z)\right)=\operatorname{Im}(z)-\operatorname{Im}(R(z))=y\left[1-\frac{n a_{n}^{2} x^{2 n-2}+\tilde{o}\left(x^{2 n-2}\right)}{a_{n}^{2} x^{2 n}+\tilde{o}\left(x^{2 n}\right)}\right] .
$$

We conclude, as before, that the quotient inside the parenthesis tends to zero when $x$ tends to $+\infty$, uniformly in $y$ for $|y| \leq 1$. Thus we can find $r_{2}$ such that

$$
\begin{aligned}
& 0<\operatorname{Im}\left(N_{F}(z)\right)<y \quad \text { if } \quad 0<y<\varepsilon \quad \text { and } \\
& y<\operatorname{Im}\left(N_{F}(z)\right)<0 \quad \text { if } \quad-\varepsilon<y<0,
\end{aligned}
$$

for all $z \in A_{r_{2}}^{\varepsilon}$.

All this together implies that if we define $r_{0}:=\max \left\{r_{1}, r_{2}\right\}$ then $N_{F}\left(A_{r_{0}+2}^{\varepsilon}\right) \subset A_{r_{0}}^{\varepsilon}$ for all $\varepsilon \in(0,1)$. Of course the result is true for all $r \geq r_{0}$.

Proof of Theorem A. Take $r:=r_{0}$ as in the proof of the previous lemma. Since $z=0$ is a superattracting fixed point, there exists $0<\varepsilon_{0}<1$ such that $B\left(0, \varepsilon_{0}\right) \subset A^{\star}(0)$. Moreover because of the immediate basin of attraction of $z=0$ is open and the interval $[0, r+2]$ is compact, there exists $0<\varepsilon_{1}<1$ so that the set $C_{r+2}^{\varepsilon_{1}}=\left\{z=x+i y|0 \leq x \leq r+2| y \mid,<\varepsilon_{1}\right\}$ lies in $A^{\star}(0)$.

From Lemma 4.4 we have $N_{F}\left(A_{r+2}^{\varepsilon_{1}}\right) \subset A_{r}^{\varepsilon_{1}}$. Moreover, from (10) we have that for all $z \in A_{r+2}^{\varepsilon_{1}}$ there exist $n>0$ such that

$$
\left(N_{F}\right)^{n}(z) \in\left(A_{r}^{\varepsilon_{1}} \backslash A_{r+2}^{\varepsilon_{1}}\right) \subset C_{r+2}^{\varepsilon_{1}} \subset A^{\star}(0) .
$$

From the previous paragraph it is clear that $B\left(0, \varepsilon_{0}\right) \cup C_{r+2}^{\varepsilon_{1}} \cup A_{r+2}^{\varepsilon_{1}}$ is a connected set belonging to $A^{\star}(0)$ with infinite area. 
Proof of Corollary 1.1. From the hypothesis, it is clear that $P(z)=\left(z-x_{0}\right)^{k} \tilde{P}_{1}(z)$ with $k$ being the multiplicity of $x_{0}$ as the (largest) real root of $P$, and $\tilde{P}_{1}$ being a polynomial of degree $m:=n-k$ with real coefficients, so that $\tilde{P}_{1}(x) \neq 0$ for all $x \geq x_{0}$. The linear change of variables $z \rightarrow z-x_{0}$ transforms $F(z)=P(z) e^{z}$ into $G(z):=F\left(z+x_{0}\right)=z^{k} P_{1}(z) e^{z}$ where $P_{1}$ is a degree $m$ polynomial with real coefficients so that $P_{1}(x) \neq 0$ for all $x \geq 0$. This proves (a).

To prove (b) we claim that $N_{G}$ and $N_{F}$ are conjugated via a linear map (sending $z=x_{0}$ to $z=0$ ). Thus, since by Theorem A applied to $N_{G}$, the immediate basin of attraction of 0 has infinite area, we will conclude that the immediate basin of attraction of $x_{0}$ for $N_{F}$ has infinite area as well.

To see the claim we write $\varphi(z)=z-x_{0}$ and notice that

$$
\begin{aligned}
\varphi \circ N_{F}(z) \circ \varphi^{-1} & =\varphi\left(N_{F}\left(z+x_{0}\right)\right)=N_{F}\left(z+x_{0}\right)-x_{0}=z+x_{0}-\frac{F\left(z+x_{0}\right)}{F^{\prime}\left(z+x_{0}\right)}-x_{0}= \\
& =z-\frac{F\left(z+x_{0}\right)}{F^{\prime}\left(z+x_{0}\right)}=z-\frac{G(z)}{G^{\prime}(z)}:=N_{G}(z) .
\end{aligned}
$$

Remark 4.2. The relaxed Newton's method applied to a smooth function $\varphi$ is defined as

$$
N_{\varphi}^{h}(z)=z-h \frac{\varphi}{\varphi^{\prime}}, \quad h \in(0,1]
$$

Under certain conditions the relaxed Newton's method may improve the convergence properties of the numerical method. It is easy to verify that the proof of Theorem A as well as Corollary 1.1 follows similarly if we substitute the Newton's method by the relaxed Newton's method.

\section{References}

[1] Beardon, A. Iteration of Rational Functions. Springer-Verlag, (1991).

[2] Çilingir, F. The Dynamics of Relaxed Newton's Method on the Exponential function and its Fractals, Thesis, Georg-August-Universität, Göttingen, (2002).

[3] Çilingir, F. Finiteness of the Area of Basins of Attraction of Relaxed Newton Method for Certain Holomorphic Functions, International Journal of Biffurcation and Chaos, Vol. 14(12), 2004, pp. 4177-4190.

[4] Çilingir, F. On infinite area for complex exponential function. Chaos, Solitons and Fractals Vol. 22, 2004, pp. 1189-1198.

[5] Haruta, M. Newton's method on the complex exponential function. Trans. Amer. Math. Soc. Vol. 351(6), 1999, pp. 2499-2513.

[6] Milnor, J. Dynamics in One Complex Variable (Third Edition), Annals of Mathematics Studies 160, Princeton University Press, 2006. 
[7] Hubbard, J., Schleicher, D., Sutherland, S. How to find all roots of complex polynomials by Newton's method. Inventiones Mathematicae, Vol. 146, 2001, pp. 1-33.

[8] Shishikura, M. The connectivity of the Julia set and fixed points. Complex Dynamics (Friends and Families), 2009, pp. 257-276. 